\title{
Risk stratification for severe acute respiratory distress syndrome requiring veno venous extracorporeal membrane oxygenation: a clinical need
}

\author{
Chiara Lazzeri, Adriano Peris \\ Emergency Department, Intensive Care Unit and Regional ECMO Referral centre, Azienda Ospedaliero-Universitaria Careggi, Florence, Italy. \\ Correspondence to: Chiara Lazzeri, MD. Emergency Department, Intensive Care Unit and Regional ECMO Referral centre, Azienda Ospedaliero- \\ Universitaria Careggi, Largo Brambilla 1, 50134 Florence, Italy. Email: lazzeric@libero.it. \\ Provenance: This is an invited article commissioned by the Editorial Board Member Prof. Federico Pappalardo (Department of Anesthesia and \\ Intensive Care, Advanced Heart Failure and Mechanical Circulatory Support Program, San Raffaele Scientific Institute and San Raffaele Vita-Salute \\ University, Milan, Italy). \\ Response to: Datzmann T, Träger K. What about prognostic outcome parameters in patients with acute respiratory distress syndrome (ARDS) treated \\ with veno-venous extracorporeal membrane oxygenation (VV-ECMO)? J Thorac Dis 2018;10:S2057-61.
}

Submitted Jul 05, 2018. Accepted for publication Jul 11, 2018.

doi: $10.21037 /$ jtd.2018.07.60

View this article at: http://dx.doi.org/10.21037/jtd.2018.07.60

The stimulating Editorial by Datzmann et al. (1) suggests further considerations on the prognostic parameters for patients on veno venous extracorporeal membrane oxygenation (VV ECMO) support. While VV ECMO is increasingly acknowledged as a life-saving therapeutic tool for refractory acute respiratory distress syndrome (ARDS) especially in high volume and experienced centers, it is also is associated with elevated costs, resource, and complications (2) and appropriate risk stratification of candidate patients is necessary to recognize those more likely to benefit from $\mathrm{VV}$ ECMO (3). Moreover, in everyday practice, an objective risk assessment for refractory ARDS on ECMO support is a clinical need for intensivists also to provide relatives with information about the patient's condition.

On a conceptual basis, a parameter holds a prognostic role when it is associated with mortality (or other adverse outcomes), independently of other variables. In this context, as elegantly pointed out by Datzmann et al. (1), lactate (measured pre-ECMO initiation, as well as 6 and 24 hours after) proved to be a prognostic factor in 126 patients with refractory ARDS treated with VV-ECMO and lactate clearance at 72 hours after ECMO initiation was independently associated with death in these patients, thus helping in the risk stratification process (4). Though the potential mechanism(s) accounting for the higher lactate levels and lower lactate clearance observed in non-survivors were only hypothesized in our paper (4), the strict relation between lactate and mostly lactate clearance and mortality represents an important clue for clinicians to monitor $\mathrm{VV}$ ECMO effects in the single patient, even in the first days after ECMO start.

Other parameters have been reported to keep a prognostic impact in VV ECMO patients. Right ventricle dilatation, observed before ECMO implantation, is one of the prognostic parameters since it was independently associated with increased mortality in 74 consecutive patients with refractory ARDS treated with VV ECMO (5). Dilatation of the right chamber is mainly determined by increased afterload, which may follow a worsening lung disease (that is disease severity, mainly hypoxic vasoconstriction, edema and atelectasia) and/or persistent increased intrathoracic pressures (indicating an aggressive ventilatory strategy, high PEEP or driving pressure). The negative impact on outcome of RV dilatation and failure is recognized in ARDS by several reports by us (5) and others (6). Body mass index (BMI) is another prognostic parameter recently reported (7-9) in patients supported with VV ECMO. Increased BMI was associated with better outcome, even if the mechanism(s) accounting for the paradoxical protective effect of increased BMI are not fully elucidated. Several hypothesis have been proposed, including more adequate nutritional score and immunomodulation by the 
adipose tissue, as well as a lower incidence of left ventricular dysfunction (7).

Still in the attempt of an efficacious risk stratification of patients with severe respiratory failure needing VV ECMO, prognostic parameters have been associated in the so called "risk scores". Unfortunately, the existing risk scores are quite heterogeneous mainly for selected population (i.e., H1N1 induced ARDS, retrieved patients), and included variables (i.e., difficult to assess such as neurological status due to deep sedation).

We therefore agree with Datzmann et al. (1) that research on prognostic parameters in ARDS patients on VV ECMO is still in his childhood mainly due to heterogeneity in the management of these patients among centers.

However, a prognostic parameter may not be a therapeutic target. While lactate (and its dynamic variations) and $\mathrm{BMI}$ are recognized prognostic parameters, they are not therapeutic goals in these patients. On the other hand, systemic inflammatory activation (as indicated by cytokine) represents a pivotal pathophysiologic mechanism of disease (as reported by Datzmann) and a therapeutic target but not a prognostic marker since, to date, cytokine levels have not be independently associated with mortality in these patients. Obviously, cytokines (and the inflammatory response) still represent a crucial topic of research in severe ARDS on VV ECMO. To make things more complex, the effect(s) of VV ECMO support itself on cytokine levels and the systemic inflammatory response are far to be completely elucidated, considering that other concomitant factors (such as antibiotic therapy, ameliorating in oxemia, $\mathrm{pH}$ and consequently systemic organ perfusion) exert a synergistic effect, though difficult to be quantified and separated.

Research in the field of VV ECMO support for respiratory failure is intriguing and challenging mainly for two reasons. Firstly because VV ECMO as a support therapy gives "time" to the patient to recover, therefore modifying the evolution/progress of the disease itself, as far as it is known. Mechanism(s) for this phenomenon as well as factors responsible for patient variability (survivors/not survivors, pulmonary fibrosis/complete lung resolution) are clinical goals of future research. Secondly, heterogeneity in the management of VV ECMO patients among centers make study results difficult to be extended from one center to another. Common research goals and protocols among different centers could overcome this difficulty.

Finally, while VV ECMO gives time to the patients to recover, disease progression urges clinicians and researchers to provide answers in quite a short time so different lines of research (clinical and experimental) should progress simultaneously taking into account that the number of patients on VV ECMO support is still increasing.

\section{Acknowledgements}

None.

\section{Footnote}

Conflicts of Interest: The authors have no conflicts of interest to declare.

\section{References}

1. Datzmann T, Trager K. What about prognostic outcome parameters in patients with acute respiratory distress syndrome (ARDS) treated with veno-venous extracorporeal membrane oxygenation (VV-ECMO)? J Thorac Dis 2018;10:S2057-61.

2. Rozencwajg S, Pilcher D, Combes A, et al. Outcomes and survival prediction models for severe adult acute respiratory distress syndrome treated with extracorporeal membrane oxygenation. Crit Care 2016;20:392.

3. Lazzeri C, Cianchi G, Mauri T, et al. A novel risk score for severe ARDS patients undergoing ECMO after retrieval from peripheral hospitals. Acta Anaesthesiol Scand 2018;62:38-48.

4. Bonizzoli M, Lazzeri C, Cianchi G, et al. Serial Lactate Measurements as a Prognostic Tool in Venovenous Extracorporeal Membrane Oxygenation Support. Ann Thorac Surg 2017;103:812-8.

5. Lazzeri C, Cianchi G, Bonizzoli M, et al. Right ventricle dilation as a prognostic factorin refractory ARDS requiring VV-ECMO. Minerva Anestesiol 2016;82:1043-9.

6. Repessé X, Charron C, Vieillard-Baron A. Acute cor pulmonale in ARDS: rationale for protecting the right ventricle. Chest 2015;147:259-65.

7. Lazzeri C, Bonizzoli M, Cianchi G, et al. Body mass index and echocardiography in refractory ARDS treated with veno-venous extracorporeal membrane oxygenation. J Artif Organs 2017;20:50-6.

8. Al-Soufi S, Buscher H, Nguyen ND, et al. Lack of association between body weight and mortality in patients on veno-venous extracorporeal membrane oxygenation. 
Intensive Care Med 2013;39:1995-2002.

9. Swol J, Buchwald D, Dudda M, et al. Veno-venous extracorporeal membrane oxygenation in obese surgical patients with hypercapnic lung failure. Acta Anaesthesiol Scand 2014;58:534-8.

Cite this article as: Lazzeri C, Peris A. Risk stratification for severe acute respiratory distress syndrome requiring veno venous extracorporeal membrane oxygenation: a clinical need. J Thorac Dis 2018;10(8):E669-E671. doi: 10.21037/ jtd.2018.07.60 